\title{
Issues of the electromagnetic current transformers searching projecting
}

\author{
Abdurauf Safarov ${ }^{1}$, Khurshid Sattarov ${ }^{2}$, Makhammatyokub Bazarov ${ }^{1}$ and Almardon \\ Mustafoqulov ${ }^{3 *}$ \\ ${ }^{1}$ Tashkent State Transport University, Tashkent, Uzbekistan \\ ${ }^{2}$ Tashkent University of Information Technologies, Tashkent, Uzbekistan \\ ${ }^{3}$ Tashkent Institute of Irrigation and Agricultural Mechanization Engineers, Tashkent, Uzbekistan
}

\begin{abstract}
The necessity of the primary transformers rapidly increases due to the fast development of the microprocessing automated control systems and operation of the railway transport power supply devices, transferring to the flexible automated output. These transformers, besides high metrology characteristics, have to be highly reliable, have a wide range of change, stability, little dimensions.
\end{abstract}

\section{Introduction}

Today, not only in our country but worldwide, microprocessor-based automated control systems are developing rapidly. The need for primary transformers is growing rapidly due to the operation of railway power supply equipment, the transition to flexible automated production. First of all, these transformers must be highly reliable, have a wide range of variability, stability, and dimensions, in addition to high metrological properties. Electromagnetic current transformers meet these requirements most of all. Increasing market demand makes producers constantly enlarge the number of issued current transformers.

\section{Methods}

According to the Research Institute of Physics Research (RIPR) [1], there exist about 400 physics phenomena nowadays, which can become ground for creating modern measure means - primary measure transformers. Some loom capacity control methods of weaving machines are known today [2]. One of the side effects of each electrical device's work is the electromagnetic field generated near its workplace. All organisms, including humans, are exposed daily to the influence of different types of this field, characterized by distinct physical parameters [3].

The most labor- and time-consuming is the initial period of project-making when it is necessary to define the primary transformer's physical principle and construction realization $[4,5]$.

\footnotetext{
* Corresponding author: mustafoali777@gmail.com
} 
Due to all this automation of the initial project period, the choice of the transformers working principles taking into account a huge amount of knowledge on physical and technical effects (PTE), is actual. These systems allow saving time and laboring while creating a new device, quickly adjusting to market change, and immediately teaching the staff.

As a result of comparative analysis of the existing automated systems of the new technical decisions search, based on work with the knowledge basics on physical effects and phenomena, there were found methods and models of physical effects describing, participating in the synthesis of technical decisions. It was defined that the most effective are the following methods and models: formalized description of natural scientific and technical effects on the ontology base of scientific and technical characteristics (Bashmakov A.I., Vladimirov A.I.), theory of decision of the inventor's tasks - the first and second generation (Altschuller G.S.), teaching CAE systems (as a sample of ILEM2001 system, worked out at Pierre and Marie Curie University (Paris, France) and Valahia (Romania), systems of the final element analysis, i.e., research of the object or process characteristics creating and deciding of the adequate mathematic model (COMSOL, Multiphysics), energy information model of chains of different physical nature and apparatus of parameter structural schemes (Zaripov M.F., Petrova I.Yu.), methods of searching construction (Polovinkin A.I., Kamaev V.A.), a combined method of the working principles search (Glazunov V.N.).

Study and comparison of the functional possibilities of these systems demonstrated that the majority of systems tend to unify, therefore at decision synthesis in them, it is impossible to apply Mathematics apparatus for calculating operating characteristics.

The energy information model of chains $[6,7,8,9]$ allows transferring to a structural formalized description of the processes in technical devices with the help of parameter structural schemes (PSS).

For combining PSS of the elements and devices of control systems, using PTE between the chains of different physical nature, they are presented as primary transformers of the quantities of the one physical nature into quantities or parameters of the other physical nature.

In the presented article, taking into consideration transformers built on two (electric and magnetic) chains, they meet the most requirements of control and operation systems. We consider some physical effects between electric and magnetic chains as well as inside each chain. They are as follows:

Physical effects between magnetic and electric chains, such as Hall's effect $\left(\mathrm{U}_{\ni}=\mathrm{Kq}_{\mu \mathrm{U}_{\ni}} \cdot \mathrm{q} \mu\right)$, effect of magnetic resistance or magnetoresistance effect $\left(\mathrm{R}_{3}=\mathrm{Kq}_{\mu \mathrm{R}} \cdot \mathrm{q} \mu\right)$, the effect of electromagnetic induction $\left(\mathrm{U}_{3}=\mathrm{K}_{\mathrm{I} \mu} \mathrm{U}_{\ni} \cdot \mathrm{I} \mu\right)$, effect of the magnetic moving power $\left(\mathrm{U} \mu=\mathrm{K}_{\mathrm{I}} \mathrm{U}_{\mu} \cdot \mathrm{I}_{\ni}\right)$; physical effects between the electric chains: Hall's effect $\left(\mathrm{U}_{\ni 1}=\mathrm{K}_{\mathrm{I}} \mathrm{U}_{\ni 1} \cdot \mathrm{I}_{\ni}\right)$,

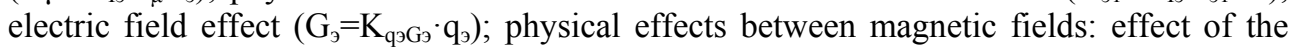
magnetic modulation at the weak magnetizing $\left(\mathrm{C} \mu \approx \mathrm{K}_{\mathrm{q} \mu 1}\right)$, at strong magnetizing $(\mathrm{W} \mu=\mathrm{Kq} \mu 2 \mathrm{w} \mu \cdot \mathrm{q} \mu 2)$.

\section{Results and Discussion}

Principles of construction of parameter structural schemes of electromagnetic current transformer (ECT) built on the dependencies mentioned above and effects are presented on graph 1. Each structural scheme, in fact, discloses this or that principle of ECT. Quantities of different physical nature, mentioned in the horizontal direction with the sign $(\approx)$, are applied to those parameters of the structural scheme, on which constant magnetic flow modulation is arranged, which is created by the transformed direct current. In the case of the alternative current transforming, the necessity of modulation disappears. 


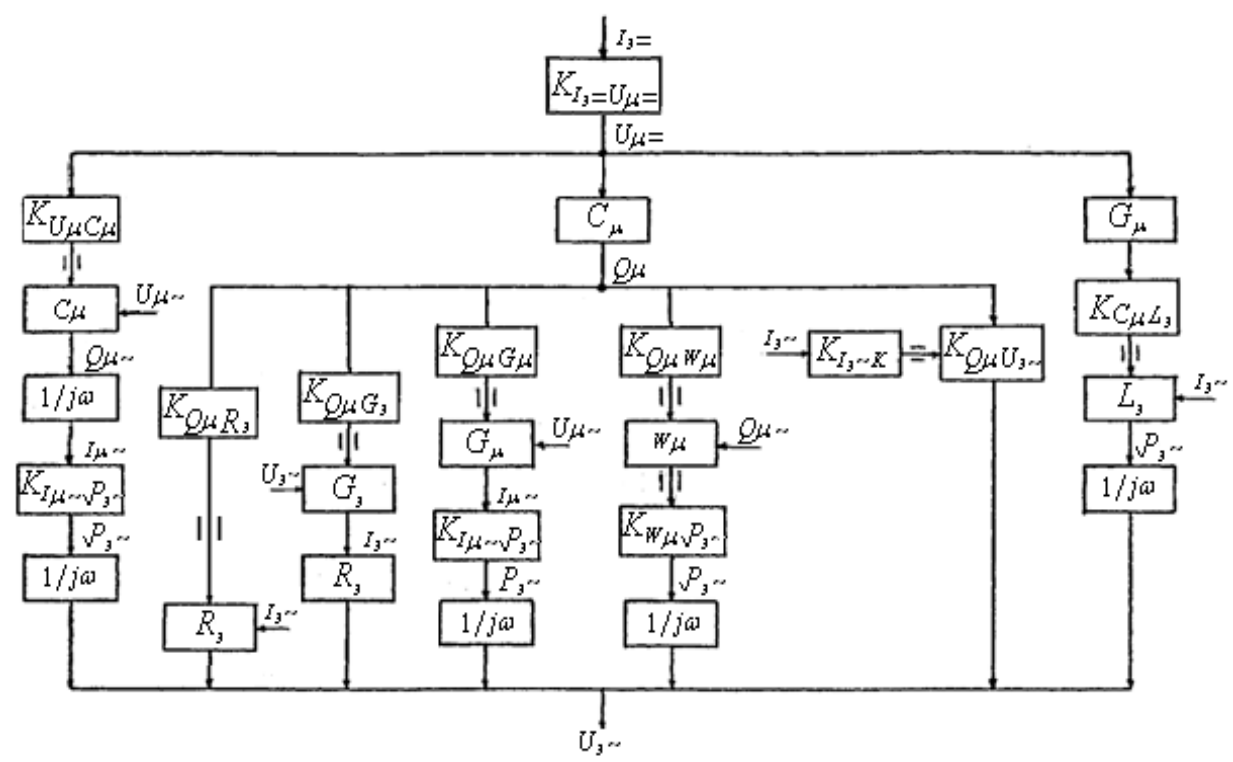

Fig.1. Principles of construction of parameter structural schemes ECT

It is important to notify that the number of structural schemes can be increased, taking into account inter-chain passages presented as topogrammes. In this case, the transformer is considered the complex of elementary chains, each of them reflects the primary transforming of one quantity into another quantity or parameter.

Coefficients of these parts transferring are either parameters of chains if relative parts reflect transformation within the chain of the same physical nature, or the coefficient of physical effects between chains if parts reflect the transformation of quantities of one physical nature into the quantity or parameter of the other physical nature.

Graphically the set of the elementary inter chain dependences is presented as a topogram. Topogram of the inter-chain dependences for the chains of different physical nature, connected with each other by graphic symbols of between chain physical effects, form general topogram of inter-chain dependences and between chain physical effects. Topograms of the inter-chain dependencies are the same for all chains of different physical nature.

It is presented in the article that from the topogram of dependences of only two different chains - electric and magnetic - the number of possible variants of the structural schemes of the electric current transformer into electric voltage is quite big.

In the case of using three chains of different nature (optic-magnetic-electric, mechanicmagnetic-electric, or nuclear-magnetic-electric), the number of possible variants of the structural scheme of the electric current transformer into electric voltage will exceed seven symbols figure.

To define most construction realizations, reflecting each PTE, method of morphology analysis and synthesis of technical decisions is chosen [10,11]. According to this method, the stage of composing the morphology matrix (MM), i.e., achieving the generalized description of the technical device all construction realizations, is called morphology analysis, and the stage of extracting the technical device construction realizations from MM is called morphology synthesis.

The morphology matrix for PTE synthesis, chosen by PSS, is presented in figure 2 .

The number of classification characteristics in it is equal to the number of PTE and parameters of PSS. The number of possible realizations of each PTE or parameter is equal 
to the number of single and pair opposite characteristics, for example, sensitivity (S), error $(\gamma)$, nonlinearity $(\varepsilon)$, sensitivity-price $(\mathrm{S}-\mathrm{P})$, error-price $(\gamma-\mathrm{P})$, and so on. Total number of possible construction realizations of ECT is equal to $\mathrm{N}=5.5 .5=125$.

Classification of the morphology characteristics notions is made with the following assumptions: 1) each morphology characteristics notion can be estimated independently from the other characteristics notions; 2) morphology characteristics have the same parameters as PTE itself. Considering these assumptions, each morphology characteristic notion is classified on the chosen characteristics on the method of pair comparison for non-strict classification.

Estimation of morphology characteristics notions method is realized as follows: on the base of pair comparison of each morphology characteristics notion with every other matrix of comparison is built $A_{i j}(i=1, n ; j=1, n)$. For each characteristic separate matrix is built.

Elements of the main diagonal are equal to zero. If $\mathrm{i}$ characteristic notion is better than $\mathrm{j}$ notion on the considered characteristic $A_{i j}=1$ in the opposite case $A_{i j}=0$. Then matrix line is built $\mathrm{Bi}$, elements of which are sums of the elements in each line, i.e.

$$
B_{i}=\sum_{i=1}^{n} A_{i j}
$$

All n elements are arranged according to the quantity $B_{i}$ On the first position is the element, where the element $B_{i}$ is maximal etc.

The next $C_{i}$ matrix is built. Elements of the matrix-line $C_{i}$ are introduced in the morphology matrix.

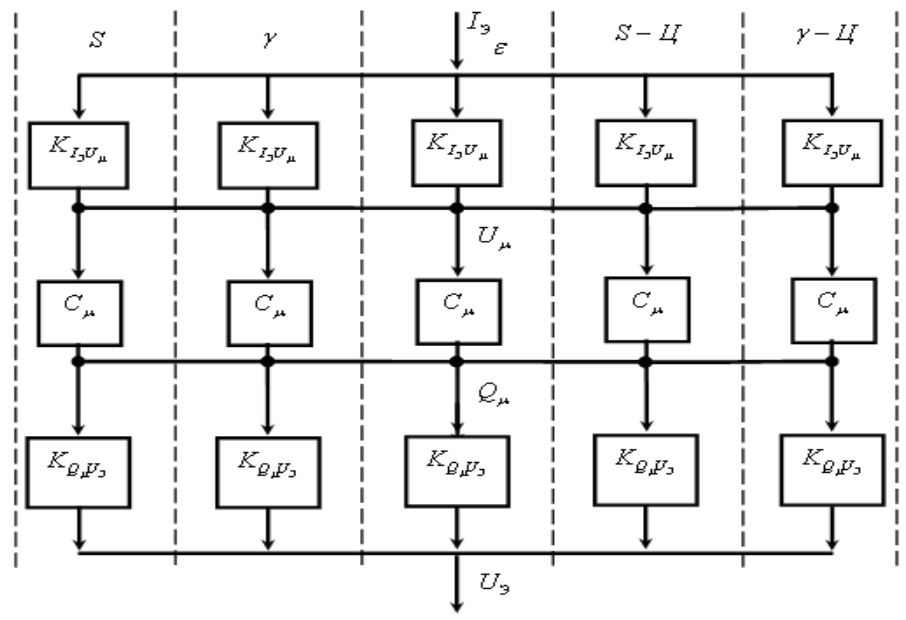

Fig. 2. Morphology matrix for PTE synthesis

For example, on the nonlinearity for morphology matrix, presented in figure 2, mentioned matrix for characteristics - PTE amper-coils have the following image: 


$$
A=\left|\begin{array}{llll}
0 & 0 & 0 & 1 \\
1 & 0 & 0 & 1 \\
1 & 0 & 0 & 1 \\
1 & 1 & 1 & 0
\end{array}\right|, B=\left|\begin{array}{l}
1 \\
2 \\
2 \\
3
\end{array}\right|, \quad C=\left|\begin{array}{l}
3 \\
2 \\
2 \\
1
\end{array}\right| .
$$

Let's assume we need the synthesis of EPT with maximal possible sensitivity. Estimation of characteristics notions is made by the method of pair comparison for nonstrict classification. The supposition is made that the variant, fixing the best notions in the morphology matrix, will be the best from the point of view of the searched goal. The characteristics notion for which sensitivity is maximal is registered on each line in every morphology matrix.

For the considered EPT, we receive the following combination of characteristics notions: implementation of PTE amper-coils - multicoil core with general winding, implementation of the magnetic capacity parameter - core with high magnetic inductivity, Hall's effect - electric magnetic induction - multicoil core with general measuring winding. As a result of morphology synthesis, we receive EPT construction [11, 12] with high sensitivity and regulated range, presented in figure 3 .

The construction consists of a metal circle, fulfilled in the form of two axes ring elements 1 and 2 with diameter connection 3 and 4, in the body at the opposite sited of which there are holes

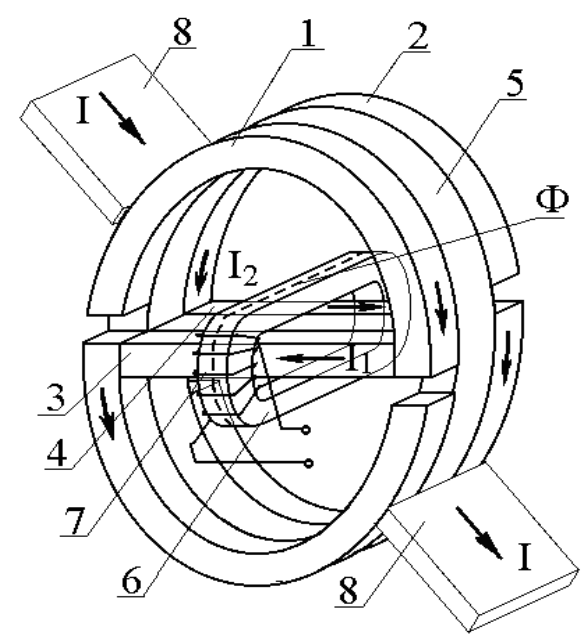

Fig.3. Construction of EPT with increased sensitivity and regulated range

$\delta_{1}$, ring elements 1 and 2 are insulated from each other by dielectric 5 and are situated in the mirror reflection, iron magnetic closed core 6 with measuring winding 7 , staged on the connections 3 and 4, situated on the opposite edges of the circle of the current receiving outputs 8 .

This implementation of the metal circle allows decreasing the sensitivity limit (to increase sensitivity) of the transformer.

EPT works on the following principle. Through the chain of the current receiving outputs 8 current under control passes. Measuring winding 7 is switched to the meter.

In the controlled chain, the maximal current of the meter range is established. Turning the metal ring in the scale, the necessary quantity of the outgoing signal is established. 
Multiple limits are arranged by creating and regulating the opposite MDC using changing the resistance of the primary winding. As with the circle turn proportionally, the arc length of bridge shoulders is changed, thus proportionally to the ankle current in the connections is changed too. Electric and magnetic forces are present practically in all-natural phenomena and determine the operation of many technical devices [13]. In addition, high magnetic fields can cause serious damage to human health. As the use of cell phones and other wireless devices is growing rapidly, this issue is more important than ever $[14,15,16,17]$. There exists a wide variety of transformer models. However, no transformer model for the study of electromagnetic transients, adequate for a wider range of frequencies, is yet available [18]. They also proposed an electromagnetic (EM) temporal model whose parameters were determined by measurements. This model is designed to describe the behavior of a transformer at high frequencies of lightning, and the voltage transmitted from the primary to the secondary is of particular interest. Therefore, he argues that the original authors could properly ignore the effects without making large errors due to nuclear saturation and hysteresis [19].

\section{Conclusion}

Thus, the combined application of the energy information and morphology method in the searching projecting to work out new construction by composing morphology matrix for each physical and technical effect of the parameter structural scheme EPT allows to sharply increase the number of synthesis variants of the current transformers and finally choose their construction with the required characteristics.

\section{References}

1. Mokrov E.A. Of 40 years of research-and-production activity of NIIFIZICHESKY researches (NIIFI), Mokrov E.A//GAUGES And SYSTEMS. № (7), pp 3-14. (2000).

2. Baratov R, Pirmatov N, Panoev A, Chulliyev Y, Ruziyev S and Mustafoqulov A. Achievement of electric energy savings through controlling frequency convertor in the operation process of asynchronous motors in textile enterprises. IPICSE 2020. IOP Conf. Series: Materials Science and Engineering1030 012161. (2021)

3. Bogdan Lewczuk, Grzegorz Redlarski, Arkadiusz Żak, Natalia Ziółkowska, Barbara Przybylska-Gornowicz and Marek Krawczuk. Influence of Electric, Magnetic, and Electromagnetic Fields on the Circadian System

4. Azimov R. K. Principles of construction and designing of primary converters with the distributed parametres for monitoring systems and management. The author's abstract dis.doctor. of tech. Sciences, TGTU,. (32) (1993).

5. Safarov A.M. Ferromagnetic converters of a direct current for control systems of electrochemical processes. Dis. Cand.Tech.Sci., Tashkent, TashPi, (1989).

6. A.M. Safarov, Kh.A. Sattarov, S.X. Jumaboyev. Device for conversion of equalizing current at the site of the traction ac network. E3S Web of Conferences 139, 01034 (2019) RSES 2019 https://doi.org/10.1051/e3sconf/201913901034, p. 3, (2019)

7. A.M. Safarov, Kh.A. Sattarov, S.X. Jumaboyev. Device for detection of the phase current asymmetry in the three-phase lines of non-traction consumers. MIST: Aerospace 2019 IOP Conf. Series: Materials Science and Engineering 734 (2020) 012196 doi: 10.1088/1757-899X/734/1/012196, p. 4. (2020). 
8. Zaripov M. F. A Method of pair comparisons at an estimation of operational characteristics of technical realisations of elements of systems of management, M.F.Zaripov, V.M.Zaripova//GAUGES And SYSTEMS. (3) pp. 31-34.( 2004).

9. Zaripov M. F., Fiks-Margolin, G. I., Vakhitova, H. Z. Analysis of different methods of approximation of the magnetization curve. - DAN UZ USSR, (3), pp. 8-10. (1974).

10. A.M. Safarov, Kh.A. Sattarov, S.X. Jumaboyev. Mathematical modeling of static characteristics of electromagnetic current converter to detect current asymmetry of the traction power supply system. International Conference on Information Science and Communications Technologies Applications, Trends and Opportunities ICISCT 2019, Tashkent, 4-6 November, 2019 http://www.icisct2019.org/ 978-1-7281-2564-0/19/ (2019)

11. Patent RUz. №03858. The current transformer / Amirov S.F., Khalikov A.A., Safarov A.M., Khushbokov B.H., Shojimov J.J., Balgaev AD//Rasmy axborotnoma, (1).( 2009).

12. Amirov S.F., Safarov A.M., Rustamov D.Sh., Ataullaev N.O. Elektromagnitnye preobrazovateli bolshix tokov dlya sistem tyagovogo elektrosnabjeniya, Tashkent, «Science and technology», p 279. (2018)

13. V.V. Denisenko. SOLUTION OF ELECTROMAGNETISM THEORY PROBLEMS. COMPUTATIONAL METHODS AND ALGORITHMS - Vol. II- Solution of Electromagnetism Theory Problems - V.V. Denisenko.

14. David O Carpenter. Human disease resulting from exposure to electromagnetic fields. PMID: 24280284 DOI: 10.1515/reveh-2013-0016. (2013).

15. Christoffer Johansen. Electromagnetic fields and health effects--epidemiologic studies of cancer, diseases of the central nervous system and arrhythmia-related heart disease. Scand J Work Environ Health.; (30). pp 1-30. (2004).

16. David O Carpenter. Electromagnetic fields and cancer: the cost of doing nothing. Rev Environ Health. Jan-Mar 2010;25(1):75-80. doi: 10.1515/reveh.2010.25.1.75. (2010),

17. Mohammed Abu-Jafar, Issam Ashqer.The Effect of the Electromagnetic Radiation from High Voltage Transformerson Students Health in Hebron Distric.

18. Francisco de Lebn, Adam Semlyen. COMPLETE TRANSFORMER MODEL FOR ELECTROMAGNETIC TRANSIENTS. IEEE Transactions on Power Delivery, ( 9), 1, January (1994)

19. F. de Leon. Discussion of "Accurate modeling of core-type distribution transformers for electromagnetic transient studies" IEEE Transactions on Power Delivery 18, (2), April (2003). 